\title{
Stimulation of the sphenopalatine ganglion in intractable cluster headache: Expert consensus on patient selection and standards of care
}

Cephalalgia

$0(0)$ I-II

(C) International Headache Society 2014 Reprints and permissions: sagepub.co.uk/journalsPermissions.nav DOI: I0.1 I77/0333/1024|4530524 cep.sagepub.com

\section{(\$)AGE}

\author{
Tim P Jürgens', Jean Schoenen², Jørgen Rostgaard ${ }^{3}$, \\ Søren Hillerup ${ }^{3}$, Miguel JA Láinez ${ }^{4}$, Alexandre T Assaf ${ }^{5}$, \\ Arne May' and Rigmor $H$ Jensen ${ }^{6}$
}

\begin{abstract}
Context and overview: Chronic cluster headache $(\mathrm{CCH})$ is a debilitating headache disorder with a significant impairment of the patients' lives. Within the past decade, various invasive neuromodulatory approaches have been proposed for the treatment of $\mathrm{CCH}$ refractory to standard preventive drug, but only very few randomized controlled studies exist in the field of neuromodulation for the treatment of drug-refractory headaches. Based on the prominent role of the cranial parasympathetic system in acute cluster headache attacks, high-frequency sphenopalatine ganglion (SPG) stimulation has been shown to abort ongoing attacks in some patients in a first small study. As preventive effects of SPG-stimulation have been suggested and the rate of long-term side effects was moderate, SPG stimulation appears to be a promising new treatment strategy.

Aims and conclusion: As SPG stimulation is effective in some patients and the first commercially available CE-marked SPG neurostimulator system has been introduced for cluster headache, patient selection and care should be standardized to ensure maximal efficacy and safety. As only limited data have been published on SPG stimulation, standards of care based on expert consensus are proposed to ensure homogeneous patient selection and treatment across international headache centres. Given that SPG stimulation is still a novel approach, all expert-based consensus on patient selection and standards of care should be re-reviewed when more long-term data are available.
\end{abstract}

\section{Keywords}

Cluster headache, sphenopalatine ganglion, stimulation, neuromodulation, standards of care, patient selection

Date received: 2 September 2013; revised: 19 December 2013; 3 February 2014; accepted: 10 March 2014

\section{Introduction}

Chronic cluster headache is an excruciating unilateral short-lasting headache mostly in the first division of the trigeminal nerve with prominent signs of ictal parasympathetic activation with lacrimation and conjunctival injection, nasal congestion and rhinorrhoea and sympathetic hypoactivity (1). It causes significant medical but also economical (2) and social impairment $(3,4)$. Although most patients respond to acute (triptans, oxygen and lidocaine) and preventative medication (verapamil, lithium, topiramate, methysergide and cortisone), a small proportion of patients are drug refractory or intolerant to established preventive drugs. For these patients, invasive (such as deep brain stimulation
'Department of Systems Neuroscience, University Medical Center Hamburg-Eppendorf, Germany

${ }^{2}$ Headache Research Unit, Department of Neurology, University of Liège, Belgium

${ }^{3}$ Department of Oral \& Maxillofacial Surgery, Copenhagen University Hospital (Rigshospitalet), Denmark

${ }^{4}$ Hospital Clinico Universitario, Catholic University of Valencia, Spain ${ }^{5}$ Department of Oral \& Maxillofacial Surgery, University Medical Center Hamburg-Eppendorf, Germany

${ }^{6}$ Danish Headache Center, Glostrup Hospital, University of Copenhagen, Denmark

\section{Corresponding author:}

Tim P Jürgens, Department of Systems Neuroscience, University Medical Center Hamburg-Eppendorf, Martinistrasse 52, D-22529 Hamburg,

Germany.

Email: t.juergens@uke.de 
of the posterior hypothalamus, occipital nerve stimulation and vagal nerve stimulation) and non-invasive neuromodulatory approaches (such as transcutaneous supraorbital nerve stimulation, transcranial direct current stimulation, transcutaneous vagal nerve stimulation and transcranial magnetic stimulation) are increasingly considered in chronic cluster headache (5-7). Although no significant difference between active stimulation with preset parameters over a period of 4 weeks compared with no stimulation could be found in the only randomized controlled cross-over trial on deep brain stimulation of the posterior hypothalamic area (8), data from the subsequent open label period along with data from open label studies and case series (8-14) have shown response rates (defined as $50 \%$ reduction of attacks) of $50 \%$ and more in chronic cluster headache. Similar response rates have been reported in open label studies of occipital nerve stimulation (15-19), whereas anecdotal reports do not allow reliable assessment of the efficacy of vagal nerve stimulation (20), high cervical spinal cord stimulation (21) and various non-invasive approaches (see $(6,7)$ for further review).

The cluster headache armamentarium has recently been augmented by stimulation of the sphenopalatine ganglion (SPG). The scientific rationale for targeting the SPG is based on the close relation between headache and autonomic activation. The clinically observed peripheral parasympathetic activation of the cranial autonomic systems with release of vasointestinal peptide (VIP) and acetylcholine during an attack is hypothesized to activate the trigeminal nociceptors in turns (22). Its effects are mediated by postganglionic parasympathetic efferents from the sphenopalatine ganglion located in the pterygopalatine fossa (PPF). The SPG receives preganglionic parasympathetic fibres from the superior salivatory nucleus in the brain stem via the greater petrosal nerve which forms the Vidian nerve together with sympathetic fibres from the carotid plexus. Although most parasympathetic postganglionic fibres synapse to postganglionic fibres in the SPG, sympathetic fibres do not. Only a few fibres from the sphenopalatine branches of the maxillary nerve enter the SPG forming the sensory root. Postganglionic parasympathetic fibres innervate the lacrimal gland, the nasopharyngeal mucous membranes (23) and meningeal vessels (24). Sensory trigeminal fibres innervate areas such as posterior nasopharynx via the lesser palatine nerve.

Upon nociceptive activation of nociceptors belonging to the first division of the trigeminal nerve in a cluster headache attack, the trigemino-parasympathetic reflex is activated. Fibres from the trigemino-cervical complex project to the superior salivatory nucleus and activate facial parasympathetic efferents (25).
As parasympathetic activation leads to vasodilation of meningeal arteries, a vicious circle further increasing trigeminal activation has been proposed. As shown in animal experiments, low-frequency SPG stimulation around $10 \mathrm{~Hz}$ can induce plasma protein extravasation as a model for neurogenic inflammation (26) and changes in blood brain barrier (27) and intracerebral vasodilation (28). In humans, low-frequency stimulation was recently shown to be able to induce attacks in cluster headache patients (29) raising the question of whether such attacks are primarily driven by a centrally mediated parasympathetic activation. Therapeutic interventions targeting the SPG have been attempted for a long time. After alcohol injection into the SPG, $85 \%$ of patients $(n=120)$ had a relevant decrease of pain (30). Other approaches include radiofrequency ablation (31) ganglionectomy (32), and - less invasive - injections of local anaesthetics (33) or corticosteroids (34) into the SPG. As most of these procedures offer only temporary benefit, and most are destructive and non-adjustable, high-frequency stimulation of the SPG has been suggested as an alternative with promising results in a single case report (35) and a case series (36). In contrast with the other neuromodulatory approaches, SPG stimulation is applied during the acute attack and not preventatively. Recently, an implantable microstimulator powered and controlled by radio frequency waves generated by an external remote controller was developed, whose electrode lead is positioned in the PPF (Autonomic Technologies, Inc., Redwood City, CA, USA). A small multicentre randomized sham-controlled study on the efficacy of acute SPG stimulation in chronic cluster headache $(n=32)$ has shown significant pain relief or pain freedom in $67 \%$ of all attacks treated with full stimulation after 15 minutes compared with subthreshold $(7 \%)$ and sham stimulation (7\%) (37). Nine of 28 patients in the experimental period $(32 \%)$ reported pain relief of $50 \%$ or more at 15 minutes stimulation in attacks with an intensity of 2 or more on a categorical rating scale (75 attacks were not considered in the final analysis as their intensity was below 2 out of 4). Of note, the latter analysis referred to a subgroup of 14 patients only, so results have to be interpreted with caution. Unexpectedly, a reduction of attack frequency of more than $50 \%$ between the baseline and the parameter adjustment period was reported in 12 out of the 28 patients $(43 \%)$ during the experimental period lasting up to 8 weeks, which seemed to be more pronounced in patients with lower attack frequencies. Although the incidence of device-related complications was low, sensory disturbances ( $81 \%$ of patients) and pain $(38 \%)$ in the territory of the maxillary nerve were observed, with the majority of these incidences being 


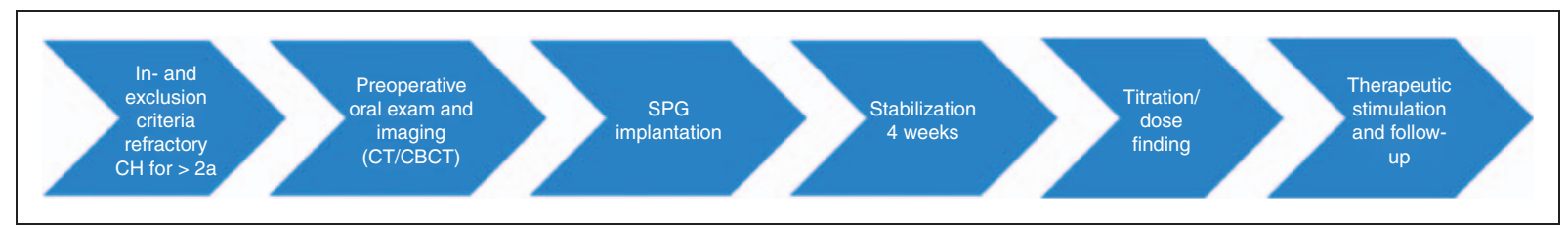

Figure I. Timeline for neurostimulator implantation in drug-refractory cluster headache $(\mathrm{CH})$ targeting the sphenopalatine ganglion (SPG). a: year; CT: computed tomography; CBCT: cone beam computed tomography.

mild and transient, as would be expected with transoral procedures of this type.

Only very few randomized controlled studies exist in the field of neuromodulation for the treatment of drugrefractory headaches and SPG stimulation may be a promising and safe alternative to the more invasive strategies in $\mathrm{CH}$ given that long-term data are congruent with these short-term observations and that these findings can be reproduced in larger samples. The SPG neurostimulator has become commercially available as a CE marked medical device in EU countries (compliant with AIMD Directive 90/385/EEC and R\&TTE Directive 1999/5/EC in 2012). The CE marking indicates that the manufacturer has verified the product's compliance with the above mentioned legislative requirements such as a harmonized level of safety, and is therefore mainly driven by safety aspects. As the ATI device is reimbursed by local healthcare providers in some countries, there is an unmet need for defining the standards of care. Therefore, we aimed to standardize patient selection and propose future longterm evaluations of its efficacy and safety in drugrefractory cluster headaches. Secondly, we aimed to make patient selection and treatment uniform across international headache centres, and therefore standards of care are proposed (see Figure 1 for timeline). In the absence of further randomized controlled studies with a special focus on long-term data, our recommendations are based on clinical experience to date and thus represent expert consensus.

\section{Preoperative management}

\section{Choice of neuromodulatory approach}

SPG stimulation is a rather recent development whose efficacy was shown in one of the very few randomized controlled studies in the field of neuromodulation for the treatment of drug-refractory headaches. However, data on efficacy and safety are so far limited to one study, unlike for occipital nerve stimulation and deep brain stimulation of the posterior hypothalamus. Choosing a neuromodulatory approach is a complex, costly and dynamic process, which may be new for many headache specialists, and influenced by factors like balance between efficacy and side effects/risks, costs, technical pros and cons, local access to the different techniques and devices, patient characteristics, regulatory and medico-legal issues, as well as recommendations by national guidelines (38). At present, no predictors of efficacy exist for any mode of neurostimulation in $\mathrm{CCH}$ which makes the decision even more difficult (for recent reviews on current neuromodulatory approaches see (5-7), for recent position paper on neuromodulation issued by the European Headache Federation see (39)).

\section{Patient selection}

Definition of refractory $\mathrm{CH}$ (adhering to published criteria). As SPG stimulation is a novel approach with no published long-term data at present and an invasive procedure, eligible patients should be medically refractory or present relative or absolute contraindications to conventional acute and/or preventive therapies even if the main intention is to use SPG stimulation to abort acute attacks only. As suggested by Goadsby and colleagues (40), this includes refractoriness to established preventatives alone and in combination exceeding the inclusion criteria of the recent controlled trial on SPG stimulation which required dissatisfaction with current treatment (37) (see (41) for current European guidelines on the treatment of cluster headache) (Table 1). Additionally, candidates should suffer from significant disability disrupting their socio-professional life. Withdrawal should be considered first if triptans and analgesics for acute medication are overused, as medication overuse headache with increased attack frequency has also been reported in patients with chronic cluster headache (for review see (42)), and other neuromodulatory approaches were less efficient in patients with medication overuse. As their application is not yet standardized, occipital nerve blocks or suboccipital injections of steroids should have been tried beforehand, based on several studies that have shown their efficacy as a preventative treatment (43-45). However, repeated application in short-time intervals may be problematic.

If interictal headaches are frequent or persist continuously, indomethacin should be given to rule out hemicrania continua and paroxysmal hemicrania. 
Table I. Definition of refractory cluster headache (modified from $(50,5 \mathrm{I}))$.

- ICHD-II criteria for cluster headache are met

- Significant interference with function or quality of life

- Failed adequate trials of preventative medication, alone or in combination (failure is defined as no therapeutic or unsatisfactory eect, intolerable side eects or contraindications to use; adequate is defined as appropriate dose and appropriate length of time)

- Consideration of medication overuse

- Failure of at least four classes (where two should come from I-3):

I. Verapamil

2. Lithium

3. Methysergide

4. Melatonin

5. Topiramate

6. Gabapentin

Table 2. Inclusion criteria for stimulation of the sphenopalatine ganglion.

Inclusion criteria

- Refractory chronic cluster headache (see Table I) with a documented headache history of at least 2 years (for patients with episodic cluster headache, see text)

- Patient suffers from a substantial number of weekly attacks

- Attacks have been documented in a diary for the recent month

- Severe psychosocial impairment (as measured by HIT-6 or MIDAS)

- Attacks are side locked or have occurred predominantly (>90\%) on the current side for the last 12 months

- Ability to distinguish cluster headaches from other headaches if concomitantly present

- Unremarkable MRI of the brain

- Implantation at a site with experience in headache diagnosis and treatment and a maxillofacial surgery team with specific training and expertise in implantation of SPG stimulators

Further inclusion and exclusion criteria. Relevant inclusion and exclusion criteria are listed in Tables 2 and 3 including the following:

a. a documented history of refractory cluster headache for at least 2 years before the implantation;

b. headache meets the current ICHD criteria for chronic cluster headache (see Use in episodic cluster headache);

c. detailed headache diary (on daily basis) for at least 1 month: number of attacks, severity and mean
Table 3. Exclusion criteria for stimulation of the sphenopalatine ganglion.

\section{Exclusion criteria}

- Lateral opening of pterygopalatine fossa is too narrow for placement of the electrode lead as confirmed on an MRI, CT or CBCT scan of the region

- Destructive or ablative procedures to the SPG with lasting deficit

- Patient has undergone facial surgery in the area of the pterygopalatine fossa or zygomaticomaxillary buttress ipsilateral to the planned implant site within the last 4 months or will undergo it in the next months

- Presence of active periodontal disease, open carious lesions, pericoronitis, dental abscess or other dental related infectious process in the ipsilateral maxillary region to be operated

- Presence of other active infectious process, such as osteomyelitis in the jaws

- Relevant osteodestructive disorder

- Malignancy in and/or radiotherapy in the facial region within the last 6 months

- Woman at childbearing age who is pregnant, breastfeeding or not using adequate contraception

- Other implantable devices using a sense amplifier, such as pacemakers or defibrillator without prior confirmation of compatibility by the manufacturer

- CSF shunt

- Withdrawal has not been considered in suspected medication overuse

- Severe psychiatric disorder other than reactive depression (such as somatoform or personality disorder)

- Serious medical condition likely requiring interventions in the facial region or frequent craniocervical MRIs (although MRI safety has been shown up to I.5 T, image quality can be impaired due to artefacts)

duration of attacks, consumption of rescue medication, circadian (and circannual) rhythmicity, response to the various preventatives including dosage, duration of intake and response;

d. attacks are side-locked or occur predominantly ( $>90 \%$ of the time) on one side. If this is not the case a bilateral approach such as bilateral occipital nerve stimulation should be considered;

e. significant disability and socio-professional impairment as measured by MIDAS (46) and/or HIT-6 (47);

f. patient is able to comprehend and comply with the instructions on how and when to use the device and will present to the headache specialist for regular follow-up visits.

It is strongly recommended to treat only patients included in either register studies or patient registries 
to avoid data loss of long-term efficacy and safety. To better assess SPG-related efficacy, patients should be able to maintain stable medication throughout the titration and therapy evaluation period. It should be noted that patients on the waiting list for implantation may remit spontaneously (9).

Use in controlled studies. To ensure comparability with other studies on the acute and/or preventive efficacy of SPG stimulation and other neuromodulatory approaches, outcome measures for future studies should be homogenous. Apart from the guidelines on the design of controlled trials published in 1995 (48), a position paper with recommendations for future studies has recently been published by the European Headache Foundation (39) paving the way for an international consensus on the design of controlled trials on neuromodulatory approaches.

Use in episodic cluster headache. As a result of a paucity of data on the efficacy of SPG stimulation in episodic $\mathrm{CH}$, no general recommendation can be given at this time. However, SPG stimulation may be indicated if the following criteria are met: the headache is well documented over the previous 2 years, refractory according to Table 1, causes high disability, occurs with several daily attacks poorly responsive to oxygen and triptans (or contraindications to use the latter because of cardiovascular conditions) or major dissatisfaction with conventional treatment, and active periods should usually last for at least 3-6 months and occur with at least yearly episodes.

Preoperative clinical oral examination. Oral examination should be conducted timely ahead of the implant procedure to exclude dental disease and other oral conditions that might compromise safe surgery. If any such conditions are found, the patient must undergo relevant dental treatment, typically sub- and supragingival scaling, correction of oral hygiene, extraction of carious teeth that cannot be restored, removal of partially erupted maxillary third molars with or without pericoronitis.

Preoperative imaging. Midfacial CT scans or cone beam computed tomography (CBCT) need to be evaluated by the implanting surgeon to:

a. ensure accessibility of the PPF;

b. exclude signs of regional infections (maxillary sinus, maxillary bone and teeth);

c. exclude signs of osteodestructive disease (such as osteoporosis or osteonecrosis);

d. exclude signs of other preexisting osseous defects (such as posttraumatic defects, dental or surgical treatment).
If any dental pathology is suspected, dental x-ray films of premolars and molars and/or panoramic views on the side to be operated should be performed preoperatively as requested by the implanting surgeon.

Patients with severe osteoporosis (especially with iatrogenic osteoporosis caused by long-term steroid treatment) or extreme maxillary atrophy should only be considered with caution as the risk for misplacement and infection may increase.

Predictive factors of success. At present, factors predictive of acute and/or preventive response to SPG stimulation are unknown. It is intriguing to assume such a predictive role for SPG blocks; however, there are no data to support such a role at present. It should be kept in mind that - despite an equally intuitive relation - occipital nerve blocks (respectively suboccipital injections of steroids) failed to show such a predictive role for effective occipital nerve stimulation.

\section{Organizational requirements including site structure}

As stated in Patient selection, sites performing implantations should have:

a. Experience in the management of patients with refractory cluster headaches and an interdisciplinary setup.

b. Surgeons with particular expertise in interventions of the mid-face, anterior skull base and infratemporal fossa (such as maxillofacial or otolaryngologist surgeons) who have undergone certified cadaver training by the company and may be assisted by visiting physicians in centres with distinct surgical expertise in SPG surgery.

c. The means to ensure long-term follow-up of implanted patients either on-site or by other qualified centres in a scientific network.

\section{Determination of implant size and target position}

Based on the preoperative CT or CBCT, a custom 3D reconstruction is provided by the manufacturer to:

a. determine implant size (approximate lengths of currently available SPG stimulators: short $3.6 \mathrm{~cm}$, medium $4.4 \mathrm{~cm}$, long $5.2 \mathrm{~cm}$, extra long $6.0 \mathrm{~cm}$ );

b. determine and visualize the target position of the electrode tip superimposed on an anterior to posterior reconstruction to guide the implanting surgeon during placement of the neurostimulator in this complex anatomical region. The target position is the putative location of the SPG usually posterior 
to the middle nasal turbinate close to the Vidian canal located in the superiomedial part of the PPF, whereas the foramen rotundum as exit point of the maxillary nerve is typically located in the superolateral PPF. The target's reference to the facial midline (lateral) and the plane of the hard palate (vertical) is recorded for later intraoperative navigation.

Current specifications for preoperative imaging are as follows: slice thickness of $0.5-1.0 \mathrm{~mm}$, reconstruction of $0.5-1.5 \mathrm{~mm}$, helical or volume acquisition, gantry tilt of $0^{\circ}$, contiguous slicing and a pitch of $1: 1$ or $1: 1.5$ (please refer to the manufacturer for more detailed and latest information).

\section{Intra- and perioperative management}

\section{Choice of anaesthesia}

The implantation procedure itself involves incision of the oral mucosa, preparation of tissue from the maxilla to place the electrode lead in the pterygopalatine fossa and fixing the stimulator body to the maxillary bone (infra-zygomatic buttress) (see Figure 2(a), part 3). Like similar operations in the field of oral and maxillofacial surgery, this can theoretically be done under local anaesthesia of the oral mucosa and the PPF.
However, positioning the device subperiostally is difficult, and some cases need repeated repositioning attempts. The procedure requires the patients to lie immobile during the implantation procedure and the fluoroscopy to prevent tissue damage to the SPG. Therefore, it is recommended to perform the implantations under general anaesthesia.

\section{Intra- or perioperative antibiotic treatment and oral decontamination}

As the neurostimulator is a foreign body inserted through the oral cavity, a single shot of an appropriate antibiotic with an extended spectrum to anaerobes (such as aminopenicillines with betalactamase inhibitors, clindamycin or newer macrolides) according to local guidelines or standards should be given preoperatively to reach sufficient levels at the beginning of the operation (49).

Oral decontamination prior to surgery should include a chlorhexidine $0.1 \%$ mouth rinse and scrubbing with swabs.

\section{Surgical procedure}

A trans-oral approach is recommended with an incision in the gingival crevice of the maxilla and appropriate relaxation incisions. A neurostimulator with an

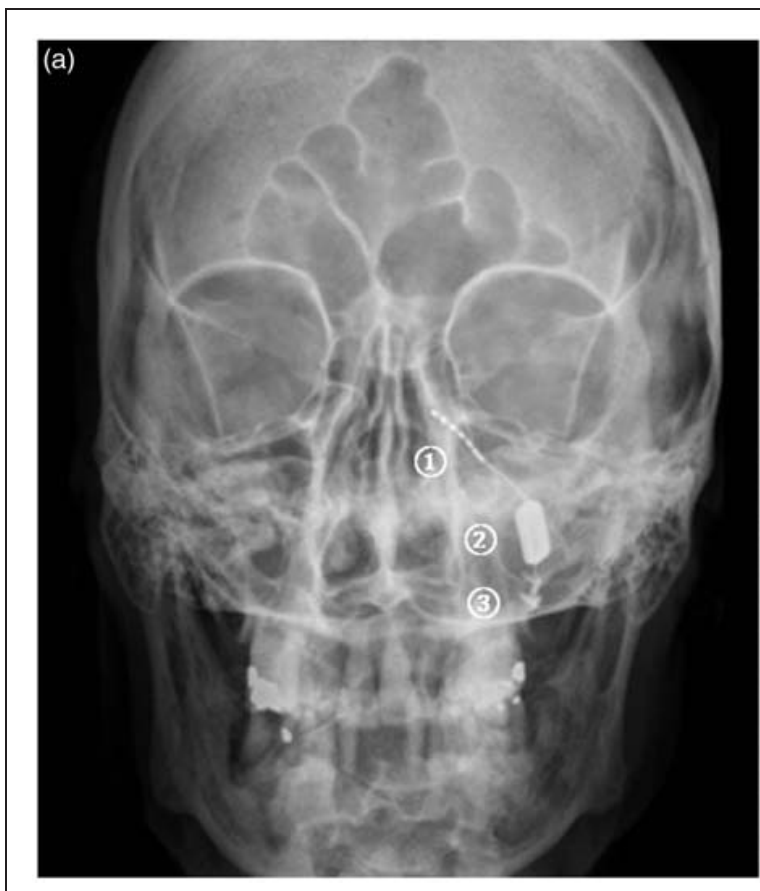

(b)

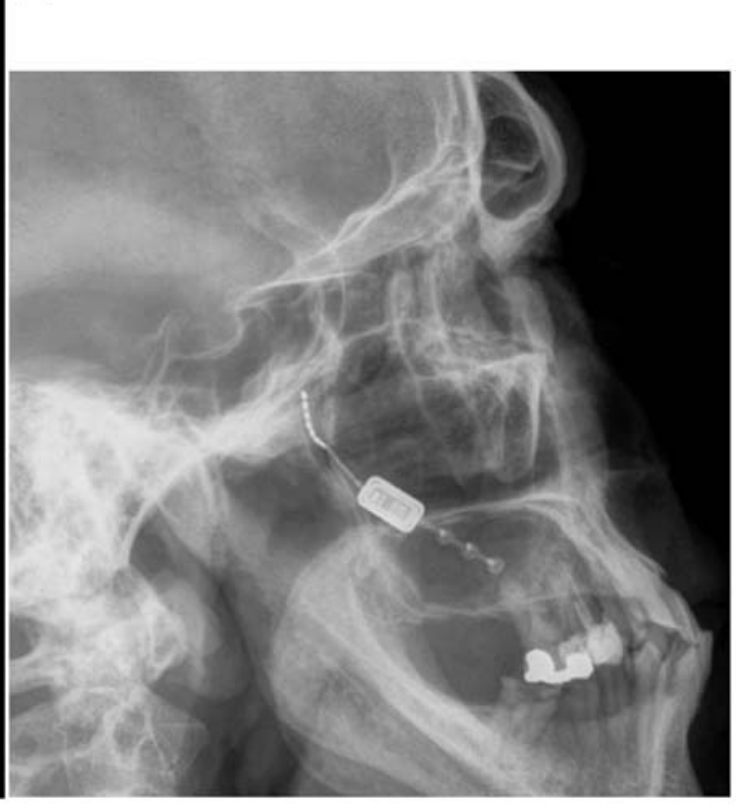

Figure 2. Sphenopalatine ganglion (SPG) neurostimulator. (a) Anterior-posterior x-ray showing the electrode lead in the pterygopalatine fossa (I), the body of the neurostimulator with the microprocessor and the RF-antenna (2) and the fixation plate (3). (b) Lateral view of SPG stimulator (modified after (6)). 
appropriate length as determined on preoperative imaging analysis of the intended implant location is inserted after blunt subperiosteal preparation of a path for the electrode lead into the pterygopalatine fossa with customized surgical tools provided by the manufacturer. Prior to insertion, the neurostimulator should be placed into a non-metallic bowl filled with ice-cold sterile water to enhance rigidity of the lead tip and, consequently, impedances should be tested at $1 \mathrm{~mA}$. The bone plate of the neurostimulator is adapted individually by bending the material. Once the correct position is confirmed by fluoroscopy or $3 \mathrm{D} x$-ray, the body of the stimulator is fixed with two or three bone screws to the zygomatic process of the maxillary bone such that the neurostimulator is placed on the posterior maxilla, medial to the zygoma and not obstructing lateral/medial excursions of the mandibular ramus (a detailed manuscript on surgical aspects of SPG implantation is under preparation) (Figure 1).

\section{Intraoperative assessment of implant position}

Imaging techniques. During blunt preparation and after placement of the neurostimulator, anterior-posterior and lateral x-rays (fluoroscopy) should be performed and compared with the target position on the reconstructed 3D image. As a result of the distortion of fluoroscopy, a reference to linear measurements (lateral to face midline on the frontal view, and cranial to the palatal plane on the lateral view) may be obtained by placing a coin or similar object with a known diameter fitted with a suture (for safe removal) in the rhinopharynx close to the film plane of the target, allowing for calculation of actual linear measurements with reasonable accuracy. No matter of projection, a coin will reflect its true diameter. After final placement of the electrode lead in the pterygopalatine fossa, the exact location should be confirmed by appropriate imaging (3D fluoroscopy is preferable over conventional anterior-posterior and lateral images as they allow a more detailed localization of the electrode lead in relation to the Vidian canal and the foramen rotundum).

Test stimulation and impedance check. As the implantation should be carried out under general anaesthesia, no mapping of paresthesias evoked by bipolar high frequency stimulation of different electrode contacts can be performed intraoperatively. The predictive value of short-term, low-frequency stimulation to provoke parasympathetic activation (28) is uncertain and no data have been published yet. After the correct position of the neurostimulator is confirmed by either fluoroscopy or $3 \mathrm{D}$ imaging and the stimulator is fixed, a final impedance check is mandatory to rule out periprocedural damage to the stimulator.

\section{Analgesic treatment of patient}

In the original publication local pain was reported perioperatively in $38 \%$ of the patients and headache in $9 \%$ (37). As most patients find it difficult to distinguish between pain caused by the implantation itself and a prolonged cluster headache attack, postoperative analgesia should pragmatically rely on both:

a. abortive medication specific for cluster headache such as sumatriptan s.c. $6 \mathrm{mg}$ (consider that injectable triptans are not available routinely in most post-anaesthesia care units) and high-flow oxygen on face masks (consider that post-op the face mask must not be held with too much pressure against the face);

b. conventional postoperative established analgesic regimes (such as paracetamol, NSAIDs alone or in combination with opioids) adhering to local standards.

\section{Pre-discharge imaging}

To safely exclude misplacement of the stimulator with the possibility of an early revision, a CT or CBCT is recommended before discharge.

\section{Post-discharge dental precautions}

Preventive and restorative dentistry can be undertaken without particular precautions except:

a. if extraction of molar teeth, root resection or other oral surgery in the operated side must be executed, this should be performed by an oral and maxillofacial surgeon who is familiar with the presence of the SPG neurostimulator;

b. local anaesthetics for dental procedures in the side of the upper jaw where the implant has been installed should be restricted to infiltrations. Block anaesthesia involving injection around the SPG neurostimulator should be avoided (tuber region).

c. the patient should rinse with chlorhexidine $0.12 \%$ twice a day in the first week. The sutures are usually removed 7-12 days postoperatively.

The patient should be provided with an implant identification card containing relevant details about the device itself and the implanting and treating physician. 


\section{Postoperative management}

\section{Stabilisation period (4 weeks)}

Wound healing is a dynamic process. Residual blood and ichor can attenuate stimulation efficacy by reducing local current density and focality due to enlarged electrical fields around the electrodes and altered impedances. As a result of ongoing resorption, electrical fields and thus stimulation parameters can vary widely, so that frequent adjustments are necessary. Unless the patient is exceptionally affected and frequent office visits are feasible, a healing period of 4 weeks is recommended. However, patients can be trained to couple their remote controller with the neurostimulator at that stage.

\section{Titration/dose finding (mostly 4-8 weeks)}

During the titration period, office visits should be performed biweekly. During the office visits:

a. impedances should be checked;

b. communication between the neurostimulator and the remote control should be checked;

c. a paresthesia and/or autonomic symptom mapping should be performed until an electrode combination is found which generates a tingling sensation in the soft palate and the root of the nose as suggested by Narouze and co-workers (30), or autonomic symptoms such as lacrimation are seen, indicating efferent activation of the parasympathetic system.

These procedures should be performed by medical personnel with special training only who should rely on medical engineering expertise (granted by the manufacturer) wherever necessary. If medico-legally acceptable, standard procedures can be delegated to a trained study nurse.

Paresthesia mapping and appropriate documentation. During paresthesia mapping, various combinations of electrodes should be tried to elicit paresthesias in the soft palate and deep in the nasopharynx. The mapping should be documented for the following parameters (electrode combination with polarity, region of paresthesia, presence of autonomic symptoms or evoked pain during test stimulation, other stimulation parameters such as frequency, amplitude and pulsewidth). Effective stimulation depends on an optimum combination of electrode contacts, the polarity of each electrode and the right stimulus settings (amplitude, frequency, pulse-width and ramp time). The choice of electrode contacts should be based on the paresthesia mapping and postoperative CT or CBCT scans. When adjusting polarity, it should be considered that current density is highest at the cathode. The following standard parameters have been used in the Pathway- $\mathrm{CH} 1$ trial as basic settings: frequency $120 \mathrm{~Hz}$, pulse width $355 \mathrm{~ms}$ and a ramp time of 20-25 seconds. The amplitude should be high enough to evoke a continuing level of paresthesias deep in the nasopharynx (30), but well below the pain threshold.

Training of patients on the use of the remote controller. As the neurostimulator is programmed and supplied with energy by a radiofrequency coil in the remote controller, continuous communication between both is critical as well. Therefore, the patient should at first be trained to identify the most effective position of the radiofrequency coil in relation to the neurostimulator. Then, the patient should learn to control the stimulation intensity by up- and downregulating the stimulation amplitude with the remote controller until the patient perceives paresthesias deep in the nasopharynx. As the device ramps the amplitude to the maximum within a predefined period, the patient should learn to adjust the amplitude so that maximum paresthesias are perceived without significant discomfort or pain. Patients should be encouraged to stimulate each attack for 15 minutes (or at least 3 minutes longer than it takes to abort attacks) with the preset parameters, and record efficacy of stimulation and side effects in the headache diary already in use.

\section{Therapeutic stimulation and follow-up}

When the stimulation parameters are optimized and acute stimulation sufficiently relieves acute attacks (or attack frequency diminishes after regular SPG stimulation), patients should be moved to the therapeutic phase. They should be encouraged to start stimulation as soon as an attack begins, as some patients report decreased efficacy of SPG neurostimulation if attacks have reached a severe or very severe intensity.

In this treatment phase, patients should be seen on a monthly basis in the first 3 months, then every 3 months for the first year. In selected cases with good response, these intervals can be stretched at an earlier time point. Telephone visits can be performed in these cases.

If SPG stimulation provides acute (and/or prophylactic) efficacy, stepwise tapering of preventative medication should not be commenced before a sustained response has been observed for at least 1-2 months. As suggested for the pre-implantation period, treatment efficacy should be continuously monitored and documented with an appropriate headache diary recording stimulation efficacy and frequency and validated tests for impairment. Preventive stimulation (stimulation outside of an attack) may also be attempted, for example for 15 minutes every morning. 


\section{MRI safety}

According to the manual provided by the manufacturer, non-clinical testing has revealed that the implantable microstimulator is MRI conditional and can be used safely in static magnetic fields of 1.5 Tesla with a spatial gradient field of 8 Tesla/meter and a maximum whole body averaged specific absorption rate (SAR) of $2 \mathrm{~W} / \mathrm{kg}$ for 15 minutes of scanning.

\section{Side effects and complications}

\section{Stimulation related side effects}

Side effects of stimulation were mostly mild and required no specific action (36). Paresthesias deep in the nasopharynx including the soft palate, which seem to be predictive of stimulation, are sometimes perceived as uncomfortable by patients. In these cases, the patients should be advised to decrease the amplitude.

\section{Implant and procedure related adverse events}

Misplacement of electrode lead. Misplacement of the electrode has been reported in three patients so far (one patient with early lead migration) (36), although this did not result in damage to surrounding structures extending the reported side effects. Red flags for misplacement are:

a. very low (can indicate excessive fluid) and very high impedance readings (indicate placement in air-filled cavities such as the maxillary sinus);

b. inability to generate adequate paresthesia in the preferred location deep in the nasopharynx;

c. missing therapeutic effect despite intense test stimulation.

In these cases, CT scans or CBCTs of the corresponding region should be obtained to ascertain lead misplacement. If the lead causes no acute potential harm in its present location, it has to be discussed with the patient whether he/she would like to simply explant the neurostimulator or wishes a reimplantation. In case of the latter, this depends on local complications (such as perforation of the lateral wall of the maxillary sinus), whether explantation and reimplantation are performed in the same session or whether a healing period of 3-6 months is necessary.

Suspected or manifest infection. In case of suspected infection, the incision site should be inspected, and imaging (CT scan, CBCT and, if necessary, bone scintigraphy) and lab tests (leucocyte count, CRP, ESR) obtained. Antibiotic treatment seems to be sufficient
(36) and should be tried before explantation of the stimulator.

No efficacy despite optimization of stimulation parameters. If no clinical benefit is achieved with acute SPG stimulation, a complete paresthesia mapping is recommended with the aim of establishing parameters that induce nasopharyngeal paresthesias. If this cannot be achieved, misplacement of the stimulation electrode or device failure should be considered. If nasopharyngeal paresthesias can be evoked, the following issues should be checked:

a. Does increasing the stimulation amplitude, the pulse width (up to 480), and/or the frequency (up to $180 \mathrm{~Hz}$ ) increase efficacy?

b. Does the patient start stimulation in the early phase of an attack?

c. Is the communication between the remote controller and the neurostimulator stable and above an average of $50 \%$ ? If not, the patient should be trained again to couple the neurostimulator with the remote controller.

d. Does the patient stimulate long enough (i.e. at least 3-5 minutes beyond resolution of the attack)?

e. Does the stimulation 'tilt' (reaching the maximum amount of available voltage) above a certain amplitude? If so, the power level of the remote controller should be adjusted.

Sensory disturbance. Sensory deficits in the distribution of V2 (including intraoral sensory deficits) were commonly reported ranging from mild hypo- to anaesthesia and resolved or improved dramatically over time (36). Because of their remitting nature, there is usually no need for further action. Further correlates of neuropathic injury include allodynia, evoked dysaesthesias and painful sensations. If not remitting or uncomfortable, they can be treated with preventative drugs used in the management of neuropathic pain, such as gabapentin, pregabaline, duloxetine and amitriptyline (50).

\section{Side-shift of attack}

Side-shifts of attacks within or between bouts occur in up to $19 \%$ of patients with cluster headache (51), although this is uncommon during an attack. It has also been reported in patients with cluster headache who received effective unilateral neurostimulation on the clinically affected side in unilateral deep brain (51) and occipital nerve stimulation (18) requiring implantation of an additional neurostimulation system on the contralateral side. In patients with a unilateral SPG stimulator and side-shift from the stimulated to the 
contralateral side, preventive drug treatment should be intensified if possible and patients should followed up for at least 12 months before an additional implant is considered on the contralateral side.

\section{Clinical implications}

- Recently, evidence has emerged that stimulation of the sphenopalatine ganglion (SPG) effectively aborts acute cluster headache attacks in some patients.

- Patient selection and patient care should be standardized to ensure maximal efficacy and safety.

- Therefore standards of care are proposed based on international expert consensus to ensure uniform patient selection and treatment across international headache centres.

\section{Funding}

This research received no specific grant from any funding agency in the public, commercial, or not-for-profit sectors.

\section{Conflict of interest}

TPJ has received honoraria for lectures and consulting from Autonomic Technologies, Inc., PharmAllergan Germany, Pfizer and MSD Germany. JS is a consultant for Autonomic Technologies, Inc, and member of the advisory boards of St Jude Medical, Medtronics, STX-Med and Allergan. JR reports no conflicts of interest. ATA and SH have been appointed as surgical consultants for ATI. ML has received honoraria and research funds from MSD, Terumo BCT, Roche, Allergan and Medtronics and is a consultant for Autonomic Technologies. AM is funded by the University Clinic of Hamburg and received unrestricted research support from LindeGas (RealFund) and scientific funds by the Deutsche Forschungsgemeinschaft (DFG), is or has been consultant or speaker for Pfizer, Bayer Vital, GSK, Allergan, ATI, MSD, and Desitin. RHJ has received honoraria for lectures and patient leaflets from MSD, BerlinChemie, ATI and Pfizer and serves on medical advisory boards for LindeGas, ATI and Neurocore.

\section{References}

1. International Headache Society, Headache Classification Committee. The International Classification of Headache Disorders, 3rd edition (beta version). Cephalalgia 2013; 33(9): 629-808.

2. Gaul C, Finken J, Biermann J, et al. Treatment costs and indirect costs of cluster headache: A health economics analysis. Cephalalgia 2011; 31(16): 1664-1672.

3. Jensen RM, Lyngberg A and Jensen RH. Burden of cluster headache. Cephalalgia 2007; 27: 535-541.

4. Jurgens TP, Gaul C, Lindwurm A, et al. Impairment in episodic and chronic cluster headache. Cephalalgia 2011; 31: 671-682.

5. Pedersen JL, Barloese M and Jensen RH. Neurostimulation in cluster headache: A review of current progress. Cephalalgia 2013; 33(14): 1179-1193.

6. Jürgens TP and Leone M. Pearls and pitfalls: Neurostimulation in headache. Cephalalgia 2013; 33(8): 512-525.

7. Magis D and Schoenen J. Advances and challenges in neurostimulation for headaches. Lancet Neurol 2012; 11(8): 708-719.
8. Fontaine D, Lazorthes Y, Mertens P, et al. Safety and efficacy of deep brain stimulation in refractory cluster headache: A randomized placebo-controlled doubleblind trial followed by a 1-year open extension. $J$ Headache Pain 2010; 11: 23-31.

9. Schoenen J, Di Clemente L, Vandenheede M, et al. Hypothalamic stimulation in chronic cluster headache: A pilot study of efficacy and mode of action. Brain 2005; 128: 940-947.

10. Leone M, Franzini A and Bussone G. Stereotactic stimulation of posterior hypothalamic gray matter in a patient with intractable cluster headache. N Engl J Med 2001; 345: 1428-1429.

11. Leone M, Franzini A, Broggi $G$ and Bussone $G$. Hypothalamic stimulation for intractable cluster headache: Long-term experience. Neurology 2006; 67: $150-152$.

12. Bartsch T, Pinsker MO, Rasche D, et al. Hypothalamic deep brain stimulation for cluster headache: Experience from a new multicase series. Cephalalgia 2008; 28: 285-295.

13. Sillay KA, Sani S and Starr PA. Deep brain stimulation for medically intractable cluster headache. Neurobiol Dis 2010; 38(3): 361-368.

14. Seijo F, Saiz A, Lozano B, et al. Neuromodulation of the posterolateral hypothalamus for the treatment of chronic refractory cluster headache: Experience in five patients with a modified anatomical target. Cephalalgia 2011; 31(16): 1634-1641.

15. Schwedt TJ, Dodick DW, Hentz J, et al. Occipital nerve stimulation for chronic headache-Long-term safety and efficacy. Cephalalgia 2007; 27: 153-157.

16. Schwedt TJ, Dodick DW, Trentman TL and Zimmerman RS. Occipital nerve stimulation for chronic cluster headache and hemicrania continua: pain relief and persistence of autonomic features. Cephalalgia 2006; 26: 1025-1027.

17. Magis D, Allena M, Bolla M, et al. Occipital nerve stimulation for drug-resistant chronic cluster headache: A prospective pilot study. Lancet Neurol 2007; 6: 314-321.

18. Magis D, Gerardy PY, Remacle JM and Schoenen J. Sustained effectiveness of occipital nerve stimulation in drug-resistant chronic cluster headache. Headache 2011; 51: 1191-1201.

19. Burns B, Watkins L and Goadsby PJ. Treatment of intractable chronic cluster headache by occipital nerve stimulation in 14 patients. Neurology 2009; 72: 341-345. 
20. Mauskop A. Vagus nerve stimulation relieves chronic refractory migraine and cluster headaches. Cephalalgia 2005; 25: 82-86.

21. Wolter T, Kiemen A and Kaube H. High cervical spinal cord stimulation for chronic cluster headache. Cephalalgia 2011; 31(11): 1170-1180.

22. Goadsby PJ and Edvinsson L. Human in vivo evidence for trigeminovascular activation in cluster headache. Neuropeptide changes and effects of acute attacks therapies. Brain 1994; 117(Pt 3): 427-434.

23. Standring S (ed.) Gray's anatomy: The anatomical basis of clinical practice, 40 edn. London: Churchill-Livingstone, 2008.

24. Uddman R, Hara H and Edvinsson L. Neuronal pathways to the rat middle meningeal artery revealed by retrograde tracing and immunocytochemistry. J Auton Nerv Syst 1989; 26(1): 69-75.

25. May A and Goadsby PJ. The trigeminovascular system in humans: Pathophysiologic implications for primary headache syndromes of the neural influences on the cerebral circulation. J Cereb Blood Flow Metab 1999; 19: 115-127.

26. Yarnitsky D, Gross Y, Lorian A, et al. Increased BBB permeability by parasympathetic sphenopalatine ganglion stimulation in dogs. Brain Res 2004; 1018(2): 236-2340.

27. Yarnitsky D, Lorian A, Shalev A, et al. Reversal of cerebral vasospasm by sphenopalatine ganglion stimulation in a dog model of subarachnoid hemorrhage. Surg Neurol 2005; 64(1): 5-11. (discussion 11.

28. Schytz HW, Barløse M, Guo S, et al. Experimental activation of the sphenopalatine ganglion provokes clusterlike attacks in humans. Cephalalgia 2013; 4(5): 416-421.

29. Devoghel JC. Cluster headache and sphenopalatine block. Acta Anaesthesiol Belg 1981; 32(1): 101-107.

30. Narouze S, Kapural L, Casanova $\mathbf{J}$ and Mekhail N. Sphenopalatine ganglion radiofrequency ablation for the management of chronic cluster headache. Headache 2009; 49(4): 571-577.

31. Meyer JS, Binns PM, Ericsson AD and Vulpe M. Sphenopalatine gangionectomy for cluster headache. Arch Otolaryngol 1970; 92(5): 475-484.

32. Yang lan $\mathrm{Y}$ and Oraee $\mathrm{S}$. A novel approach to transnasal sphenopalatine ganglion injection. Pain Physician 2006; 9(2): 131-134.

33. Felisati G, Arnone F, Lozza P, et al. Sphenopalatine endoscopic ganglion block: a revision of a traditional technique for cluster headache. Laryngoscope 2006; 116: 1447-1450.

34. Ibarra E. Neuromodulación del ganglio esfenapalatino para aliviar los síntomas de la cefalea en racimos. Reporte de un caso. Rev Iberoamericana del Dolor 2006; 1: $10-17$.

35. Ansarinia M, Rezai A, Tepper SJ, et al. Electrical Stimulation of Sphenopalatine Ganglion for Acute Treatment of Cluster Headaches. Headache 2010; 50: 1164-1174.

36. Schoenen J, Jensen RH, Lantéri-Minet M, et al. Stimulation of the sphenopalatine ganglion (SPG) for cluster headache treatment. Pathway CH-1: A randomized, sham-controlled study. Cephalalgia 2013; 33(10): 816-830.

37. Jürgens TP, Paulus W, Tronnier V, et al. Einsatz neuromodulierender Verfahren bei primären Kopfschmerzen Therapieempfehlungen der Deutschen Migräne und Kopfschmerzgesellschaft. Nervenheilkunde 2011; 30: 47-58.

38. Goadsby PJ, Schoenen J, Ferrari MD, et al. Towards a definition of intractable headache for use in clinical practice and trials. Cephalalgia 2006; 26(9): 1168-1170.

39. May A, Leone M, Afra J, et al. EFNS guidelines on the treatment of cluster headache and other trigeminal-autonomic cephalalgias. Eur J Neurol 2006; 13: 1066-1077.

40. Paemeleire K, Evers S and Goadsby PJ. Medicationoveruse headache in patients with cluster headache. Curr Pain Headache Rep 2008; 12: 122-127.

41. Afridi SK, Shields KG, Bhola R and Goadsby PJ. Greater occipital nerve injection in primary headache syndromes-prolonged effects from a single injection. Pain 2006; 122: 126-129.

42. Ambrosini A, Vandenheede M, Rossi P, et al. Suboccipital injection with a mixture of rapid- and long-acting steroids in cluster headache: a double-blind placebo-controlled study. Pain 2005; 118: 92-96.

43. Leroux E, Valade D, Taifas I, et al. Suboccipital steroid injections for transitional treatment of patients with more than two cluster headache attacks per day: a randomised, double-blind, placebo-controlled trial. Lancet Neurol 2011; 10: 891-897.

44. Stewart WF, Lipton RB, Whyte J, et al. An international study to assess reliability of the Migraine Disability Assessment (MIDAS) score. Neurology 1999; 53: 988-994.

45. Kosinski M, Bayliss MS, Bjorner JB, et al. A six-item short-form survey for measuring headache impact: the HIT-6. Qual Life Res 2003; 12(8): 963-974.

46. Dent CD, Olson JW, Farish SE, et al. The influence of preoperative antibiotics on success of endosseous implants up to and including stage II surgery: a study of 2,641 implants. J Oral Maxillofac Surg 1997; 55(12 Suppl 5): 19-24.

47. Attal N, Cruccu G, Baron R, et al. EFNS guidelines on the pharmacological treatment of neuropathic pain: 2010 revision. Eur J Neurol 2010; 17(9): e1113-e88.

48. Bahra A, May A and Goadsby PJ. Cluster headache: a prospective clinical study with diagnostic implications. Neurology 2002; 58: 354-361.

49. Leone M, Franzini A, Broggi G, et al. Long-term followup of bilateral hypothalamic stimulation for intractable cluster headache. Brain 2004; 127: 2259-2264.

50. Goadsby PJ, Schoenen J, Ferrari MD, et al. Towards a definition of intractable headache for use in clinical practice and trials. Cephalalgia 2006; 26: 1168-1170.

51. Leone M, May A, Franzini A, et al. Deep brain stimulation for intractable chronic cluster headache: proposals for patient selection. Cephalalgia 2004; 24: 934-937. 